\title{
Peripherality of breakup reactions
}

\author{
P. Capel *1, 田 and F. M. Nunes 2 , 用 \\ ${ }^{1}$ TRIUMF, 4004 Wesbrook Mall, Vancouver, B.C., Canada V6T2A3 \\ ${ }^{2}$ National Superconducting Cyclotron Laboratory and Department of Physics and Astronomy, \\ Michigan State University, East Lansing, Michigan 48824, USA
}

(Dated: September 13, 2018)

\begin{abstract}
The sensitivity of elastic breakup to the interior of the projectile wave function is analyzed. Breakup calculations of loosely bound nuclei $\left({ }^{8} \mathrm{~B}\right.$ and $\left.{ }^{11} \mathrm{Be}\right)$ are performed with two different descriptions of the projectile. The descriptions differ strongly in the interior of the wave function, but exhibit identical asymptotic properties, namely the same asymptotic normalisation coefficient, and phase shifts. Breakup calculations are performed at intermediate energies (40-70 MeV/nucleon) on lead and carbon targets as well as at low energy $(26 \mathrm{MeV})$ on a nickel target. No dependence on the projectile description is observed. This result confirms that breakup reactions are peripheral in the sense that they probe only the external part of the wave function. These measurements are thus not directly sensitive to the total normalization of the wave function, i.e. spectroscopic factor.
\end{abstract}

PACS numbers: 24.10.-i, 25.60.Gc, 27.20.+n

Keywords: Coulomb dissociation, asymptotic normalization coefficient, spectroscopic factor, $A=11, A=8$

\section{INTRODUCTION}

The development of radioactive ion beams in the mid-80s has allowed the study of nuclei far from stability. This technical breakthrough led to the discovery of halo nuclei on the neutron-rich side of the valley of stability [1, 2]. These loosely bound nuclei have a strongly clusterized structure [3, 4, 5, 6]. In a simple model, they are seen as a core, that contains most of the nucleons, to which one or two neutrons are loosely bound. Due to this small binding, the valence neutrons tunnel far outside the classically allowed region and form a sort of halo around the core [7]. Although less probable, proton halos are also possible.

Being very short lived, these nuclei can be studied only by indirect techniques like elastic breakup. In this reaction, the halo dissociates from the core through interaction with a target [3, 4, 5, 6]. From the comparison of the data with the theoretical prediction, one usually extracts a spectroscopic factor for the single-particle configuration of the projectile model [8, 9, 10, 11]. Alternatively, it has been suggested that breakup reactions being highly peripheral, only the asymptotic normalization coefficient (ANC) can be extracted from the measurements [12, 13]. These two viewpoints are contradictory and must be disentangled.

Recent theoretical papers show that breakup calculations are sensitive mainly to asymptotic properties of the projectile [14, 15, 16]. Both the ANC of the initial bound state, and the phase shifts in the continuum play significant roles in the dissociation. However, they do not prove the breakup not to be sensitive to the interior of the bound-state wave function.

In most reaction studies, peripherality of a reaction refers to insignificant contributions to the breakup cross section from small distances between the projectile and the target (e.g. [17, 18]). However here we are interested in peripherality relative to the internal coordinate of the projectile, the distance between the core and the valence nucleon. This notion of peripherality is intimately related to the sensitivity of the reaction to the interior of the projectile wave function. Since it is not directly related to the projectile-target peripherality, it deserves particular attention.

To analyze the role played by the internal part of the wave function in the dissociation, we perform breakup calculations using two descriptions of the projectile that differ only in the interior of the wave function. To obtain such a pair of descriptions, we use the supersymmetric transformations developed by Baye [19, 20]. Their great advantage is that the initial Hamiltonian and its supersymmetric partner exhibit identical asymptotic properties. Therefore, both descriptions exhibit the same phase shifts and ANC, but differ strongly in the interior.

\footnotetext{
* Present address: Physique Quantique, C.P. 165/82 and Physique Nucléaire Théorique et Physique Mathématique, C.P. 229, Université Libre de Bruxelles (U.L.B.), B-1050 Brussels, Belgium

${ }^{\dagger}$ Electronic address: pierre.capel@centraliens.net

${ }^{\ddagger}$ Electronic address: nunes@nscl.msu.edu
} 
The difference between calculations performed using both descriptions will tell us about the sensitivity to the interior of the projectile wave function. In this study, we consider two projectiles: a proton-halo nucleus $\left({ }^{8} \mathrm{~B}\right)$, and a neutronhalo nucleus $\left({ }^{11} \mathrm{Be}\right)$. The calculations are performed at intermediate $(40-70 \mathrm{MeV} /$ nucleon $)$ and low $(26 \mathrm{MeV})$ energies using either the dynamical eikonal model of breakup [21, 22] or the Continuum Discretized Coupled Channel technique (CDCC) 23, 24]. We also consider Coulomb and nuclear dominated reactions. In each case, the differences between both descriptions are analyzed through different observables (energy, angular, and parallel-momentum distributions).

In Sec.II. we present the projectile descriptions used in the calculations, including a summary on the supersymmetric transformations. The results of our analysis are displayed in Sec. III] The conclusions are drawn in the last section.

\section{PROJECTILE DESCRIPTIONS}

\section{A. Supersymmetric transformations}

In both the dynamical eikonal [21, 22], and the CDCC [23, 24] models of breakup reactions, the projectile is described as a two-body system: a spherical, structureless core $c$ to which a pointlike fragment $f$ is loosely bound. The internal structure of the projectile is modeled by the Hamiltonian

$$
H_{0}=-\frac{\hbar^{2}}{2 \mu} \Delta+V_{0}(\boldsymbol{r})
$$

where $\boldsymbol{r}$ is the relative coordinate of the fragment to the core, and $\mu$ is the reduced mass of the $c$ - $f$ system. The potential $V_{0}$ describes the interaction between the core and the fragment. It is adjusted to reproduce the loosely bound states, and some of the resonances of the projectile. It contains both Coulomb and nuclear terms. For the present study, we choose $V_{0}$ deep enough to obtain an additional deeply bound state in the partial wave of the physical ground state. This spurious state has no physical meaning, and merely serves to create a node in the interior of the wave function of the loosely bound state.

To obtain a second description of the projectile, that differs from the first only in the interior, we remove the spurious deeply bound state. This eliminates the node in the wave function of the physical ground state. However, we want to preserve the ANC of the bound state as well as the phase shifts obtained with $V_{0}$, because they play a major role in breakup calculations [14, 15, 16]. As shown by Baye [19, 20], this can be done using a pair of supersymmetric transformations. The first removes the deeply bound state, without altering the other levels of the spectrum [25]. The second restores the phase shifts of $V_{0}$ that are modified by the first [19, 20]. The resulting potential $V_{2}$ exhibits the same spectrum as the initial $V_{0}$ but for the removed state. It is also phase equivalent as it gives the same phase shifts. Moreover, the ANC of the remaining bound states are identical in both descriptions.

The supersymmetric elimination is performed only in the partial wave of the removed state. Therefore, $V_{2}$ depends strongly on the angular momentum even if initially a single potential is chosen to describe all partial waves. In the $l j$ partial wave, the new potential reads [20]

$$
V_{2}^{l j}(r)=V_{0}^{l j}(r)-\frac{\hbar^{2}}{\mu} \frac{d^{2}}{d r^{2}} \ln \int_{0}^{r}\left[u_{l j}^{0}\left(E_{0}, r^{\prime}\right)\right]^{2} d r^{\prime},
$$

where $u_{l j}^{0}$ is the reduced radial wave function of the removed state of energy $E_{0}$. The potential $V_{2}$ differs from $V_{0}$ only in the range of $u_{l j}^{0}$. Similarly, the wave functions of the Hamiltonian eigenstates are affected only at short distances. After the supersymmetric elimination, the wave functions read [20]

$$
u_{l j}^{2}(E, r)=u_{l j}^{0}(E, r)-u_{l j}^{0}\left(E_{0}, r\right) \frac{\int_{0}^{r} u_{l j}^{0}\left(E_{0}, r^{\prime}\right) u_{l j}^{0}\left(E, r^{\prime}\right) d r^{\prime}}{\int_{0}^{r}\left[u_{l j}^{0}\left(E_{0}, r^{\prime}\right)\right]^{2} d r^{\prime}},
$$

where $u_{l j}^{0}$ and $u_{l j}^{2}$ are the reduced radial wave functions obtained with $V_{0}$ and $V_{2}$, respectively, for either bound or scattering states. The asymptotic properties of $V_{0}$ (ANC of the remaining bound states, and phase shifts in the continuum) are therefore preserved by these transformations.

\section{B. Descriptions of ${ }^{8} \mathrm{~B}$ and ${ }^{11} \mathrm{Be}$}

Our description of ${ }^{8} \mathrm{~B}$ is based on the two-body model used in Refs. [24, 26]. The parameters of the ${ }^{7} \mathrm{Be}-p$ potential are the same as in those former papers but for the depth of the central term in the $p 3 / 2$ partial wave. For that 


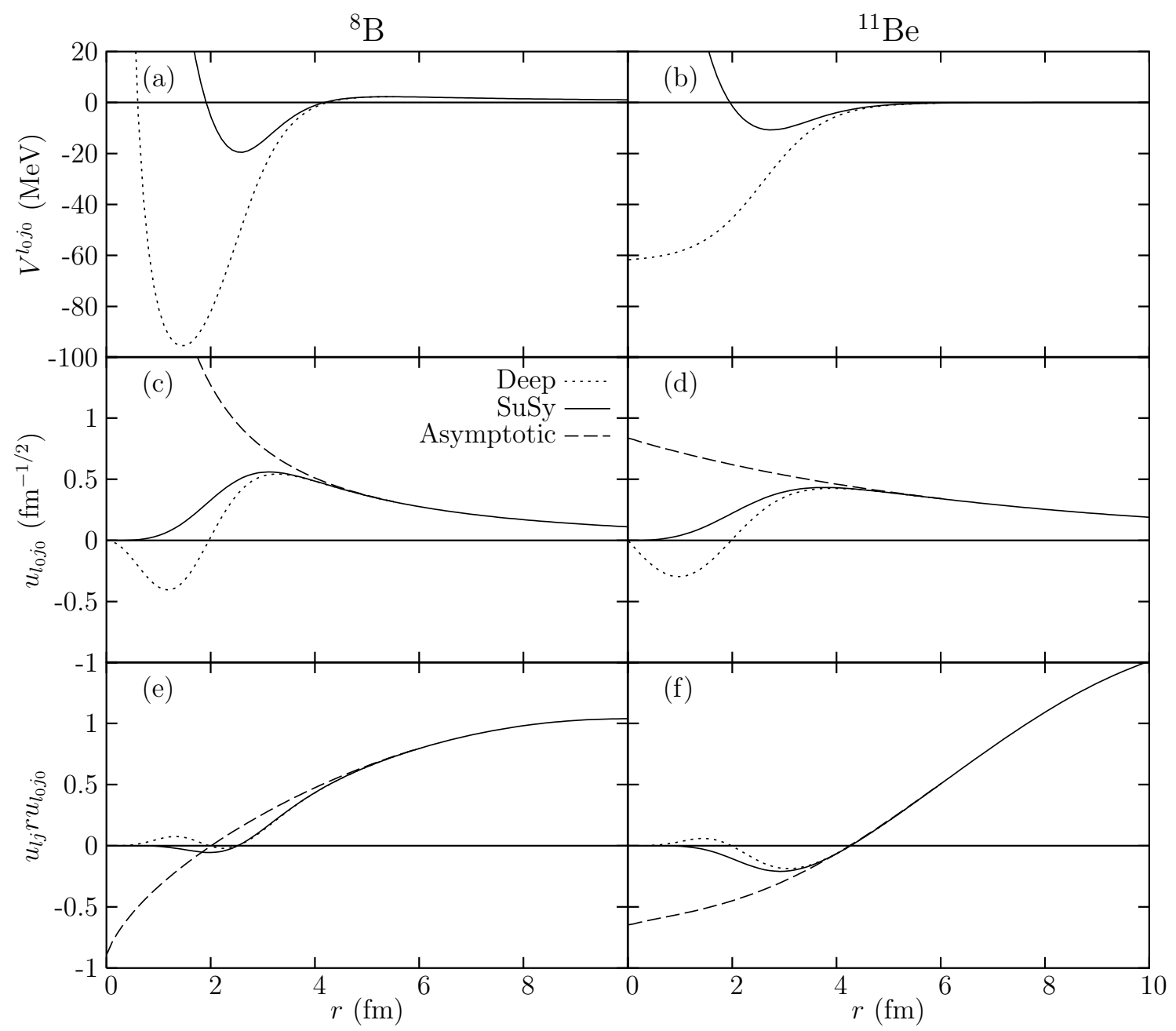

FIG. 1: Effective potentials describing the core-fragment interaction for ${ }^{8} \mathrm{~B}$ (a), and ${ }^{11} \mathrm{Be}$ (b). Both the initial deep potentials (dotted lines), and their supersymmetric partners (full lines) are depicted. Radial wave functions of the loosely bound state of ${ }^{8} \mathrm{~B}(\mathrm{c})$, and ${ }^{11} \mathrm{Be}(\mathrm{d})$. Their asymptotic behavior extrapolated down to $r=0$ is also shown (dashed line). Radial integrand appearing in the first-order approximation of the transition from the ground state to a continuum state at $E=1 \mathrm{MeV}$ in the $s 1 / 2$ partial wave for ${ }^{8} \mathrm{~B}(\mathrm{e})$, and in the $p 3 / 2$ partial wave for ${ }^{11} \mathrm{Be}(\mathrm{f})$ (see Sec. IIIB).

partial wave we consider a much deeper Woods-Saxon form factor, which reproduces not only the loosely bound state of ${ }^{8} \mathrm{~B}$ but also a spurious deeply bound state (at $E_{0 p 3 / 2}=-57.2 \mathrm{MeV}$ ). This potential (including the Coulomb and centrifugal terms), and the corresponding radial wave function of the physical ground state are displayed in Figs. 1(a) and (c), respectively (dotted lines).

To obtain the second description of the nucleus, we remove the spurious $p 3 / 2$ state using the supersymmetric transformations (see Sec. IIA). This gives us the new potential in the $p 3 / 2$ partial wave plotted as a full line in Fig. 1(a). The corresponding wave function of the physical $p 3 / 2$ state is shown in Fig. 1(c) (full line). As expected, both wave functions are identical above $4 \mathrm{fm}$, where they coincide with their asymptotic behavior (dashed line). However, they strongly differ in the interior, where one exhibits a node, while the other is nodeless.

To describe ${ }^{11} \mathrm{Be}$, we consider the same ${ }^{10} \mathrm{Be}-n$ potential as in Ref. [27]. This model includes a Pauli forbidden state in the $s 1 / 2$ partial wave of the physical ground state $\left(E_{0 s 1 / 2}=-32.7 \mathrm{MeV}\right)$. To obtain the second ${ }^{11}$ Be description, we simply remove that state using the supersymmetric transformations. The initial (dotted line) and supersymmetric (full line) potentials are displayed in Fig. 1(b). The wave functions of the physical loosely bound state are displayed in Fig. 1(d) for both descriptions. As in the ${ }^{8} \mathrm{~B}$ case, they differ at small distance, while they concur above 4 fm. 


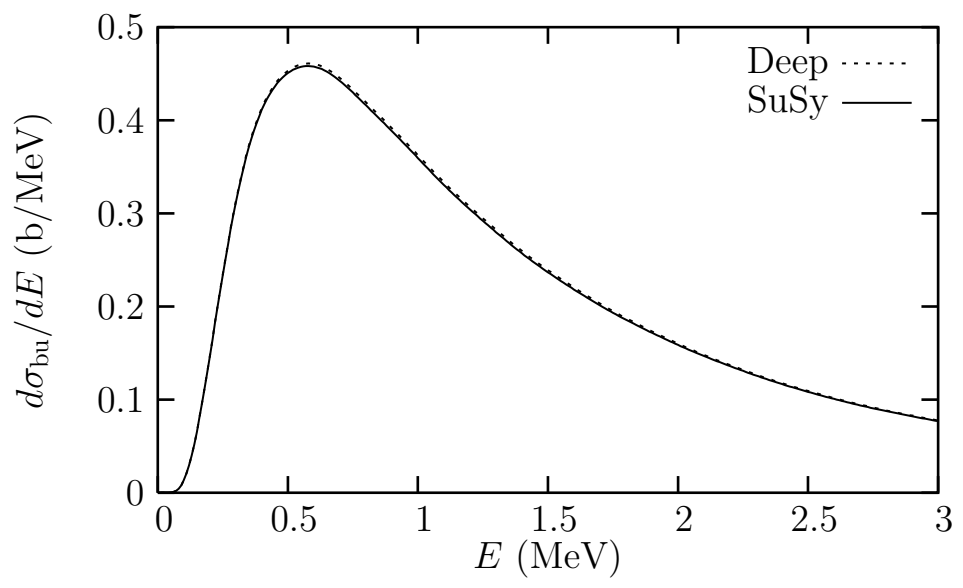

FIG. 2: Breakup cross section of ${ }^{8} \mathrm{~B}$ on $\mathrm{Pb}$ at $44 \mathrm{MeV} /$ nucleon as a function of the energy between the proton and the ${ }^{7} \mathrm{Be}$ after dissociation. The results are obtained using either the deep potential (dotted line) or its supersymmetric partner (full line).

\section{BREAKUP CALCULATION}

\section{A. Coulomb breakup at intermediate energy}

Using the two descriptions of ${ }^{8} \mathrm{~B}$, we perform calculations of its breakup on lead at $44 \mathrm{MeV} /$ nucleon. It corresponds to the experiment performed at MSU by Davids et al. [28]. For this study, we use the dynamical eikonal approximation [21, 22]. To simulate the interactions between the projectile components and the lead target, we follow Mortimer et al. [26].

The results of the calculations are illustrated in Fig. 2, where the breakup cross section is plotted as a function of the relative energy between the ${ }^{7} \mathrm{Be}$ core and the proton after dissociation. The difference between the two calculations is completely negligible (about $0.5 \%$ ). This confirms that breakup probes only the tail of the projectile wave function, since it is not sensitive to the large differences in the interior.

A partial wave analysis of this cross section shows that the same result is obtained for the contribution of all partial waves. The tiny difference observed in Fig. 2 is not due to some accidental cancellation. Negligible differences are also observed in angular and parallel-momentum distributions. Note that the presence of the spurious deeply bound state in the $p 3 / 2$ partial wave of the deep potential does not affect the breakup calculation. The cross section for the inelastic process leading to the population of this state is indeed of $8 \mu \mathrm{b}$, which is five orders of magnitude below the total breakup cross section ( $0.874 \mathrm{~b}$ for the deep potential, and $0.869 \mathrm{~b}$ for its supersymmetric partner).

The present result is in perfect agreement with the analysis presented in Ref. [29]. In that paper, it was found that eliminating the forbidden bound states from the ${ }^{11}$ Be spectrum has a very weak effect on the calculation of its breakup on lead at $70 \mathrm{MeV} /$ nucleon.

\section{B. First-order analysis}

With the aim of understanding qualitatively this result, we make a first-order analysis. In this approximation [30], the breakup is assumed to occur in one step from the initial bound state to the continuum. The reaction being Coulomb dominated, we consider here only the Coulomb interaction, which we expand into multipoles. The breakup cross section can then be expressed analytically as the sum of the contributions of the multipoles [30]. The same result is obtained within this framework: the difference between both descriptions is negligible.

The dependence on the projectile description of the contribution of the multipole $E \lambda$ comes from the radial integral

$$
\mathcal{R}_{E l j}^{(\lambda)}=\int_{0}^{\infty} u_{l j}(E, r) r^{\lambda} u_{l_{0} j_{0}}\left(E_{0}, r\right) d r
$$

where $u_{l j}$ is the radial wave function describing the $c$ - $f$ continuum in the $l j$ partial wave at energy $E$, and $u_{l_{0} j_{0}}$ is the radial wave function of the initial bound state. The integrand in Eq. (4) is displayed in Fig. 11(e) for the dominant $E 1$ transition from the initial $p 3 / 2$ bound state of ${ }^{8} \mathrm{~B}$ to the $s 1 / 2$ continuum state computed at $E=1 \mathrm{MeV}$. This 


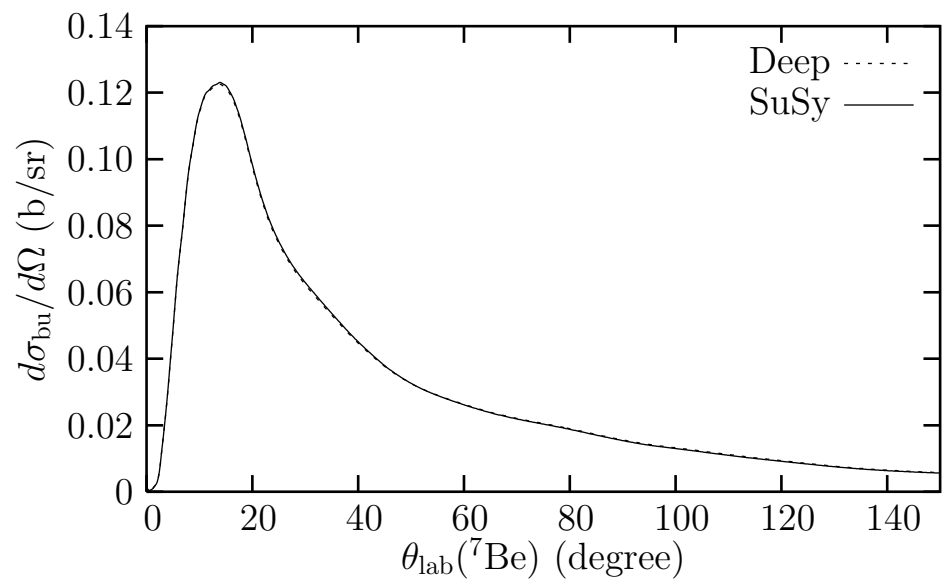

FIG. 3: Breakup cross section of ${ }^{8} \mathrm{~B}$ on $\mathrm{Ni}$ at $26 \mathrm{MeV}$ as a function of the ${ }^{7} \mathrm{Be}$ scattering angle after dissociation. The results are obtained using either the deep potential (dotted line) or its supersymmetric partner (full line). The difference is so small that both curves are superimposed.

integrand for the $E 1$ transition in ${ }^{11} \mathrm{Be}$ from the $s 1 / 2$ bound state to the $p 3 / 2$ continuum state at $E=1 \mathrm{MeV}$ is depicted in Fig. 1(f). For both nuclei, the major contribution to $\mathcal{R}_{E l j}$ comes from radii above 4 fm, where the wave functions obtained with the deep and supersymmetric potentials coincide. The interior of the wave functions contributes very little to the integral mainly because of the large tail of the wave function of the loosely bound state. Moreover, the vanishing behavior of the radial wave functions near the origin, multiplied by the $r^{\lambda}$ factor, strongly reduces the contribution of the interior. This explains qualitatively why the internal part of the wave function has nearly no effect on breakup calculations. It also indicates that one cannot choose any form factor of the wave functions in the interior. For example, one could use the extrapolation down to $r=0$ of the asymptotic behavior of the wave functions [see dashed lines in Figs. 1(c) and (d)]. This leads to the dashed curves in Figs. 1(e) and (f). In this case, the interior contribution is obviously overemphasized. If non-vanishing wave functions are used, it is necessary to resort to a cutoff at low radius, as Typel and Baur in Ref. [15].

\section{Coulomb breakup at low energy}

To see if the result of Sec. IIIA holds at lower energy, we calculate the breakup of ${ }^{8} \mathrm{~B}$ on nickel at $26 \mathrm{MeV}$. It corresponds to the experiment performed at Notre Dame [31]. This reaction has been analyzed within the CDCC framework in Ref. [24]. For the present study, the calculations are performed in a similar way using the code FRESCO $32]$.

The angular distribution of the ${ }^{7}$ Be core after breakup is shown in Fig. 3 . The difference between both ${ }^{8} \mathrm{~B}$ descriptions is even smaller than in the previous case: the two results are indistinguishable. Interestingly this is true for the entire angular range. In particular, even at large scattering angle, where the nuclear interaction dominates, no difference can be observed between both ${ }^{8} \mathrm{~B}$ descriptions. The same conclusions are drawn from the energy distribution.

This additional result strengthens the conclusion of Sec. III A by extending its validity toward low energies. The dissociation of loosely bound nuclei therefore never seems to reflect the interior of the projectile. Only the tail of the wave function is probed by such reactions.

\section{Nuclear-dominated breakup}

Finally, we investigate the influence of the interior of the projectile wave function onto nuclear dominated reactions. We choose the breakup of ${ }^{11} \mathrm{Be}$ on carbon at $68 \mathrm{MeV} /$ nucleon. This reaction has been measured at RIKEN by Fukuda et al. [11]. It has been successfully described using the dynamical eikonal approximation [22, 27], as well as the CDCC technique [33]. We use the former model with the same inputs as in Ref. [27].

The breakup cross section is plotted in Fig. 4 as a function of the relative energy between the ${ }^{10} \mathrm{Be}$ core and the neutron after dissociation. The sensitivity to the potential choice is similar to the previous calculations. Both descriptions lead to nearly identical cross sections. The same conclusion can be drawn from angular and parallelmomentum distributions. This result shows that even in nuclear-dominated breakup, the calculations are not sensitive 


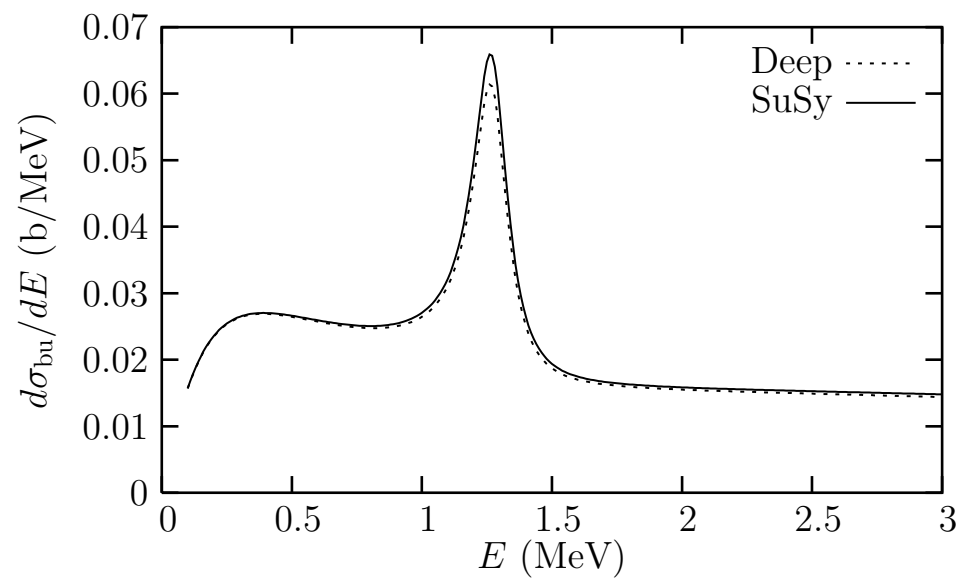

FIG. 4: Breakup cross section of ${ }^{11} \mathrm{Be}$ on $\mathrm{C}$ at $68 \mathrm{MeV} /$ nucleon as a function of the energy between the neutron and the ${ }^{10} \mathrm{Be}$ after dissociation. The results are obtained using either the deep potential (dotted line) or its supersymmetric partner (full line).

to the internal part of the projectile wave function. As in the previous cases, only the tail of the wave function is probed.

The only place where the two descriptions give slightly different results is in the peak at $1.3 \mathrm{MeV}$, where they differ by $8 \%$. The peak is due to the presence of a $\frac{5}{2}^{+}$resonance at that energy [27]. Since the difference appears only in the $d 5 / 2$ partial wave, where the resonance is reproduced, we attribute this effect to the particular behavior of the continuum wave function. This wave function exhibits a huge peak near the origin due to the resonant nature of the state. In a first-order viewpoint, this peak increases significantly the contribution of the interior to the radial integral of Eq. (44). Consequently, a larger difference is observed. This effect is in agreement with the analysis of Ref. [29], where it has been observed that the excitation to the $\frac{1}{2}^{-}$bound state of ${ }^{11} \mathrm{Be}$ is more sensitive than the breakup to the removal of the Pauli forbidden states. In that case, both the initial and final states are bound. As in the present case, the low- $r$ contribution is enhanced. Therefore, due to the shorter range of the final-state wave function, the excitation to bound or resonant states seems more sensitive to the interior of the wave function than the non-resonant breakup.

\section{E. Comparison with projectile-target peripherality}

As mentioned in the introduction, other theoretical studies refer to peripherality of reactions as insensitivity to small projectile-target distances [17, 18]. For compact projectiles, no contributions from small impact parameters implies the insensitivity to the internal part of the projectile wave function. However, this may not be the case for loosely bound projectiles and reaction processes with multi-step mechanisms.

Up to now, we have focused on peripherality relative to the internal coordinate of the projectile. In this section, we analyse the sensitivity of the breakup probability $P_{\mathrm{bu}}$ as a function of the impact parameter $b$. In semiclassical models, this breakup probability corresponds to the contribution of one trajectory to the total breakup cross section. This is illustrated in Fig. 5 .

The upper part (a) corresponds to the ${ }^{8} \mathrm{~B}$ breakup on lead at $44 \mathrm{MeV} /$ nucleon for a relative energy $E=0.5 \mathrm{MeV}$ between the ${ }^{7} \mathrm{Be}$ core and the proton after dissociation. The breakup probability computed using the deep potential is displayed in dotted line. The full line corresponds to the relative difference between the results obtained with the deep potential and its supersymmetric partner. The first important point to notice is that the reaction occurs at very large impact parameters: most of the contributions occur beyond impact parameter of $15 \mathrm{fm}$. Secondly, in the region where there are contributions to the cross section, there is less than $1 \%$ difference between the calculations using the deep projectile potential and the supersymmetric partner, confirming no sensitivity to the projectile wave function in the interior. This is a case where the reaction is peripheral relative to both, projectile-target and core-fragment distances. However, the two peripheralities seem disconnected. Beyond $15 \mathrm{fm}$, the relative difference between the deep and supersymmetric potentials is indeed very constant. It does not decrease with $b$ : the sensitivity to the interior of the projectile description is the same at large projectile-target distances $(b \sim 100 \mathrm{fm})$ than at intermediate ones $(b \sim 15 \mathrm{fm})$. 


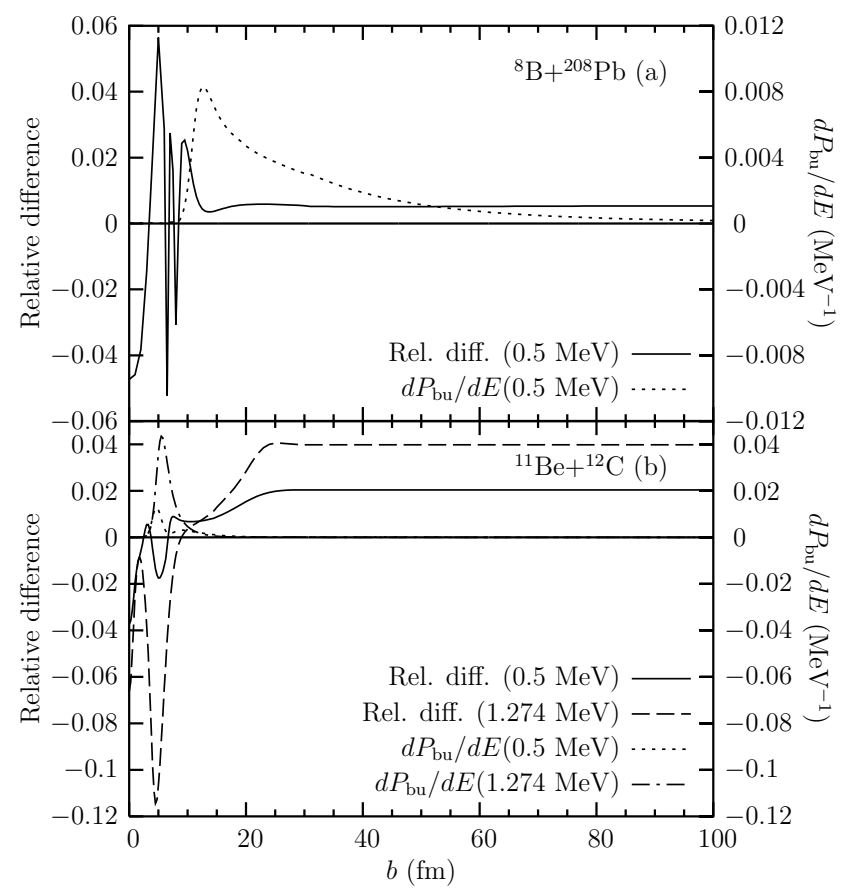

FIG. 5: Relative difference between the deep and supersymmetric calculations in the breakup probability as a function of the impact parameter. The breakup probability obtained with the deep potential is shown as well. (a) Breakup of ${ }^{8} \mathrm{~B}$ on $\mathrm{Pb}$ at $44 \mathrm{MeV} /$ nucleon for $E=0.5 \mathrm{MeV}$. (b) Breakup of ${ }^{11} \mathrm{Be}$ on $\mathrm{C} 68 \mathrm{MeV} /$ nucleon for $E=0.5 \mathrm{MeV}$, and $E=1.274 \mathrm{MeV}$ (i.e. $\frac{5}{2}^{+}$-resonance energy).

We perform the same analysis for the dissociation of ${ }^{11} \mathrm{Be}$ on carbon at $68 \mathrm{MeV} /$ nucleon. The breakup probability, and relative difference between the two descriptions are displayed in Fig. [5(b) for two relative energies $E$ : $0.5 \mathrm{MeV}$, and $1.274 \mathrm{MeV}$, the energy of the $\frac{5}{2}^{+}$resonance. In this case, the dominant contribution to the breakup cross section comes from impact parameters around $b=5 \mathrm{fm}$. Expectedly, this nuclear dominated dissociation is less peripheral than Coulomb breakup. However, the difference between both descriptions remains negligible, except for the resonance energy, as seen in Sec. IIID. Therefore, even though the reaction appears to be surface peaked in the projectile-target distance, it is still insensitive to the description of the projectile at small core-fragment distances.

\section{CONCLUSION}

In this paper, the sensitivity of elastic breakup to the internal part of the projectile wave function is analyzed. We compare calculations of dissociation of loosely bound nuclei performed with two descriptions of the projectile, which differ only in the interior of the wave function. These descriptions are obtained from phase equivalent potentials constructed with supersymmetric transformations [19, 20].

We start from an initial deep potential adjusted to reproduce, not only the loosely bound state of the projectile, but also a spurious deeply bound state. Due to the presence of that unphysical state, the projectile wave function exhibits a node near the origin. A second description is obtained by removing the spurious state. The wave function therefore no longer exhibits a node. This removal is achieved by supersymmetric transformations, which preserve the asymptotic properties of the initial potential [19, 20] (i.e. ANC and phase shifts). This enables us to analyze the sensitivity of the calculations only to the interior of the wave function.

We study this effect in the dissociation of two halo nuclei: ${ }^{8} \mathrm{~B}$, and ${ }^{11} \mathrm{Be}$. Both Coulomb and nuclear induced reactions are considered, as well as intermediate and low energy regimes. In all the analyzed cases, no difference between the deep potential and its supersymmetric partner is obtained, although the corresponding wave functions of the projectile strongly differ in the interior (presence or absence of a node). This is observed in several observables such as energy, angular, and parallel-momentum distributions. We conclude that the elastic breakup of loosely bound nuclei is peripheral in the sense that it is not sensitive to the interior of the description of the projectile. Only the asymptotic properties of the projectile model are probed through these reactions; namely the ANC of the bound state, as suggested in Refs. [12, 13], and the phase shifts in the continuum [14, 15, 16]. Therefore, two descriptions 
of the projectile corresponding to the same ANC but different spectroscopic factors will lead to the same theoretical prediction. This shows that spectroscopic factors extracted from breakup measurements will contain an error due to the imprecision of the projectile description at short distances, which is not probed by breakup reactions. The extraction of an ANC from breakup is less model dependent. Nevertheless, it will still contain uncertainties associated with the projectile-target interactions and the description of the projectile continuum.

\section{Acknowledgments}

We thank J. A. Tostevin for interesting discussions on this topic. This work has been initiated during the program INT-05-3 organized by the Institute for Nuclear Theory at the University of Washington. P. C. thanks this institute for its hospitality and the Department of Energy for partial support during the program. He also acknowledges the support of the Natural Sciences and Engineering Research Council of Canada (NSERC). Support from the National Superconducting Cyclotron Laboratory (NSCL) at Michigan State University and from the National Science Foundation grant PHY-0456656 is acknowledged.

[1] I. Tanihata, H. Hamagaki, O. Hashimoto, S. Nagamiya, Y. Shida, N. Yoshikawa, O. Yamakawa, K. Sugimoto, T. Kobayashi, D. E. Greiner, N. Takahashi, and Y. Nojiri, Phys. Lett. B 160, 380 (1985).

[2] I. Tanihata, H. Hamagaki, O. Hashimoto, Y. Shida, N. Yoshikawa, K. Sugimoto, O. Yamakawa, T. Kobayashi, and N. Takahashi, Phys. Rev. Lett. 55, 2676 (1985).

[3] P. G. Hansen, A. S. Jensen, and B. Jonson, Ann. Rev. Nucl. Part. Sci 45, 591 (1995).

[4] I. Tanihata, J. Phys. G 22, 157 (1996).

[5] B. Jonson, Phys. Rep. 389, 1 (2004).

[6] G. Baur, K. Hencken, and D. Trautmann, Prog. Part. Nucl. Phys. 51, 487 (2003).

[7] P. G. Hansen, B. Jonson, Europhys. Lett. 4, 409 (1987).

[8] T. Nakamura, S. Shimoura, T. Kobayashi, T. Teranishi, K. Abe, N. Aoi, Y. Doki, M. Fujimaki, N. Inabe, N. Iwasa, K. Katori, T. Kubo, H. Okuno, T. Suzuki, I. Tanihata, Y. Watanabe, A. Yoshida, and M. Ishihara, Phys. Lett. B 331, 296 (1994).

[9] R. Palit, P. Adrich, T. Aumann, K. Boretzky, B. V. Carlson, D. Cortina, U. D. Pramanik, T. W. Elze, H. Emling, H. Geissel, M. Hellström, K. L. Jones, J. V. Kratz, R. Kulessa, Y. Leifels, A. Leistenschneider, G. Münzenberg, C. Nociforo, P. Reiter, H. Simon, K. Sümmerer, and W. Walus, Phys. Rev. C 68, 034318 (2003).

[10] U. D. Pramanik, T. Aumann, K. Boretzky, B. V. Carlson, D. Cortina, T. W. Elze, H. Emling, H. Geissel, A. Grnschloss, M. Hellström, S. Ilievski, J. V. Kratz, R. Kulessa, Y. Leifels, A. Leistenschneider, E. Lubkiewicz, G. Münzenberg, P. Reiter, H. Simon, K. Sümmerer, E. Wajda, and W. Walus, Phys. Lett. B 551, 63 (2003).

[11] N. Fukuda, T. Nakamura, N. Aoi, N. Imai, M. Ishihara, T. Kobayashi, H. Iwasaki, T. Kubo, A. Mengoni, M. Notani, H. Otsu, H. Sakurai, S. Shimoura, T. Teranishi, Y. X. Watanabe, and K. Yoneda, Phys. Rev. C 70, 054606 (2004).

[12] L. Trache, F. Carstoiu, C. A. Gagliardi, and R. E. Tribble, Phys. Rev. Lett. 87, 271102 (2001).

[13] L. Trache, F. Carstoiu, C. A. Gagliardi, and R. E. Tribble, Phys. Rev. C 69, 032802(R) (2004).

[14] S. Typel, G. Baur, Phys. Rev. Lett. 93, 142502 (2004).

[15] S. Typel, G. Baur, Nucl. Phys. A759, 247 (2005).

[16] P. Capel, F. M. Nunes, Phys. Rev. C 73, 014615 (2006).

[17] G. Baur, F. Rösel, D. Trautmann, and R. Shyam, Phys. Rev. 111, 333 (1984).

[18] R. Shyam, H. Lenske, Phys. Rev. C 57, 2427 (1998).

[19] D. Baye, Phys. Rev. Lett. 58, 2738 (1987).

[20] D. Baye, J. Phys. A 20, 5529 (1987).

[21] D. Baye, P. Capel, and G. Goldstein, Phys. Rev. Lett. 95, 082502 (2005).

[22] G. Goldstein, D. Baye, and P. Capel, Phys. Rev. C 73, 024602 (2006).

[23] M. Kamimura, M. Yahiro, Y. Iseri, H. Kameyama, Y. Sakuragi, and M. Kawai, Prog. Theor. Phys. Suppl. 89, 1 (1986).

[24] J. A. Tostevin, F. M. Nunes, and I. J. Thompson, Phys. Rev. C 63, 024617 (2001).

[25] C. V. Sukumar, J. Phys. A 18, 2917 (1985).

[26] J. Mortimer, I. J. Thompson, and J. A. Tostevin, Phys. Rev. C 65, 064619 (2002).

[27] P. Capel, G. Goldstein, and D. Baye, Phys. Rev. C 70, 064605 (2004).

[28] B. Davids, S. M. Austin, D. Bazin, H. Esbensen, B. M. Sherrill, I. J. Thompson, and J. A. Tostevin, Phys. Rev. C 63, 065806 (2001).

[29] P. Capel, D. Baye, and V. S. Melezhik, Phys. Lett. B 552, 145 (2003).

[30] K. Alder, A. Winther, Electromagnetic Excitation (North-Holland, Amsterdam, 1975).

[31] J. J. Kolata, V. Guimarães, D. Peterson, P. Santi, R. H. White-Stevens, S. M. Vincent, F. D. Becchetti, M. Y. Lee, T. W. O'Donnell, D. A. Roberts, and J. A. Zimmerman, Phys. Rev. C 63, 024616 (2001).

[32] I. J. Thompson, Comput. Phys. Rep. 7, 167 (1988). 
[33] D. J. Howell, J. A. Tostevin, and J. S. Al-Khalili, J. Phys. G 31, S1881 (2005). 